\title{
Heterogeneidade Neuropsicológica na Esclerose Múlipla
}

\author{
Neuropsychological Heterogeneity in Multiple Sclerosis
}

\author{
Eduardo de Paula Lima* ${ }^{*}$, Vitor Geraldi Haase ${ }^{b} \&$ Marco Aurélio Lana-Peixoto $^{b}$ \\ ${ }^{a}$ Faced - Faculdade de Ciências Econômicas, Administrativas e Contábeis de Divinópolis, Divinópolis, Brasil \\ ${ }^{b}$ Universidade Federal de Minas Gerais, Belo Horizonte, Brasil
}

\begin{abstract}
Resumo
A esclerose múltipla (EM) é uma doença neurológica progressiva e incapacitante, cujos sintomas afetam o funcionamento motor, sensorial e psicológico. Diversas variáveis clínicas e psicossociais influenciam o perfil neuropsicológico na EM, que é extremamente heterogêneo. O objetivo principal do presente estudo foi investigar a possibilidade de estratificar estatisticamente portadores de EM e controles a par tir das dimensões neurológica, sócio-demográfica e neuropsicológica de funcionamento. A par tir desse objetivo, foi conduzido um procedimento de análise de conglomerados utilizando cinco instrumentos neuropsicológicos, selecionados dentre uma gama de nove medidas, de acordo com a acurácia diagnóstica. A amostra foi constituída por 45 pessoas saudáveis e 35 portadores de EM com características sócio-demográficas semelhantes. Os resultados indicaram uma solução ideal com quatro conglomerados a partir de duas dimensões, funcionamento "cognitivo" e "psicossocial", que representam aspectos independentes, porém não disjuntivos, do funcionamento neuropsicológico na EM.

Palavras-chave: Esclerose múltipla; avaliação neuropsicológica; validade de critério; análise de conglomerados.
\end{abstract}

\begin{abstract}
Multiple sclerosis (MS) is a progressively disabling neurological disease which symptoms affect sensory, motor and psychological functioning. Several clinical neurological and psychological variables influence the neuropsychological profile in MS, which is extremely heterogeneous. The main objective of the present study was to investigate if it is possible to statistically stratify control subjects and MS patients from neurological, socio-demographic and neuropsychological dimensions. With this purpose we applied cluster analysis procedures to five neuropsychological instruments selected according to diagnostic accuracy from a pool of 9 neuropsychological tests. The sample was composed of 45 healthy controls and 35 MS patients with similar socio-demographic characteristics. The results indicated an ideal solution with 4 different clusters according to two dimensions: "cognitive" and "psychosocial" functioning, which represent independent but non-disjunctive aspects of neuropsychological functioning in MS.

Keywords: Multiple Sclerosis; neuropsychological assessment; criteria validity; cluster analysis.
\end{abstract}

A esclerose múltipla (EM) é uma das doenças neurológicas crônicas e incapacitantes que comprometem adultos jovens e de meia idade com maior freqüência em todo o mundo (Rao, 1986; Thornton \& Raz, 1997; Wishart \& Sharpe, 1997; Zakzanis, 2000). A causa da EM é desconhecida, mas os modelos etiológicos postulam a interação de mecanismos genéticos e ambientais, provavelmente uma infecção viral adquirida antes da puberdade, na origem do quadro clínico (O'Connor, 2002). Os mecanismos lesionais consistem tanto de episódios de desmielinização no sistema nervoso central, causados por um processo inflamatório perivascular autoimune, quanto al terações axonais progressivas de causa genético-degenerativa e/ou agravados pelos episódios prévios de desmielinização (O’Connor).

A doença é muito heterogênea, sob diversos aspectos. Três formas clínicas principais são distinguidas (Lublin \& Reingold, 1996). Na forma recorrente-remitente (RR)

\footnotetext{
* Endereço para correspondência: Rua Sebastião Possada Bravo, 105/202, Bairro Santa Rosa, Belo Horizonte, MG, 31255-760. E-mail: edpl@hotmail.com
}

predominam os episódios de desmielinização, que podem cursar com recuperação parcial ou completa dos sintomas entre os surtos. Após alguns anos de evolução, muitos pacientes com a forma RR evoluem para uma forma secundária progressiva (SP), em que começam a ocorrer lesões por degeneração axonal e a doença assume um curso progressivo independentemente da ocorrência de surtos. Finalmente, alguns pacientes apresentam uma forma primária progressiva (PP) da doença, na qual predominam, desde o início, as manifestações degenerativo-axonais e o curso é progressivo.

Tanto as manifestações clínicas quanto o cur so evolutivo da doença são imprevisíveis para um paciente individual. As manifestações variam desde comprometimentos motores, sob a forma de paralisias, tremor ou incoordenação motora, alterações sensoriais, manifestas por anestesia, parestesias, dor etc., até al terações emocionais e cognitivas (McIntosh-Michaelis et al., 1991). As alterações emocionais predominantes são a ansiedade e o estresse relacionados com o curso imprevisível da doença, bem como os sintomas de depressão maior, que parece ter uma base 
neurobiológica adicional à reatividade emocional pela incapacidade (Pujol et al., 2000). O comprometimento cognitivo é, geralmente, moderado, sendo raros os casos de demência. Mas devido a sua prevalência, da ordem de 30\% em estudos transversais, tanto os comprometimentos afetivo-emocionais quanto cognitivos apresentam um impacto considerável sobre o bem-estar subjetivo, adaptação familiar e funcionamento ocupacional dos portadores (Gainotti, 2006).

O perfil neuropsicológico na EM é bastante heterogêneo. Os domínios mais freqüentemente comprometidos são o funcionamento executivo, a memória episódica e a função motora das mãos (Rao, 1986; Zakzanis, 2000). Do ponto de vista psicossocial, além dos sintomas de estresse e de depressão, que já foram mencionados, a fadiga é sintoma prevalente em mais de $2 / 3$ dos pacientes, o que agrava potencialmente as incapacidades observadas (O'Connor, 2002). Fatores como idade, escolaridade, funcionamento neurológico, idade de início e duração da doença, e forma clínica da esclerose múltipla são discutidos como variáveis preditoras do desempenho dos pacientes (O'Connor). Contudo, estudos metanalíticos têm apontado resultados controversos a esse respeito (Thornton \& Raz, 1997; Wishart \& Sharpe, 1997; Zakzanis, 2000).

A heterogeneidade clínica da esclerose múltipla pode ser exacerbada em função dos mecanismos de neuroplasticidade e regeneração funcional. Utilizando-se de técnicas de neuroimagem funcional, Rocca et al. (2005) obtiveram evidências de reorganização cortical durante a execução de gestos em pacientes com EM. Pacientes com graus maiores de deficiências neurológicas apresentaram padrões mais amplos de ativação cortical durante a execução de gestos. Um mesmo paciente pode, portanto, apresentar lesões em diferentes estágios de evolução/regeneração. Diferentes pacientes apresentam, por outro lado, potenciais distintos para recuperação funcional. Evidências clínicas sugerem, inclusive, que alguns pacientes podem ter um curso relativamente benigno, apresentam uma carga lesional cumulativa mínima e, portanto, uma virtual ausência de incapacidades associadas à doença (Amato, Zipoli, Goretti et al., 2006).

A heterogeneidade fisiopatogênica e clinica constatada na EM reflete-se no contexto da avaliação neuropsicológica. Até cerca de dois terços dos pacientes com EM recrutados na comunidade podem não apresentar comprometimentos neuropsicológicos em estudos transversais (Amato, Zipoli, \& Portaccio, 2006). No mais longo estudo longitudinal conduzido sobre déficits cognitivos na esclerose múltipla foi observado que apesar de incialmente $74 \%$ dos pacientes não apresentarem déficits cognitivos significativos, esta percentagem se reduziu para 44\% após 10 anos de seguimento (Amato, Zipoli, \& Portaccio, 2006). Os dados quanto ao prognóstico cognitivo da EM indicam, portanto, que um diagnóstico neuropsicológico preciso é de fundamental importância clinica na previsão do curso evolutivo da doença.

As dificuldades enfrentadas na avaliação neuropsicológica de portadores de EM são compostas pela necessi- dade de utilizar um conjunto de testes flexível, adequado às correlações estrutura-função típicas da doença, com acurácia alta, adequado às características dos pacientes, abrangendo as principais funções mentais e, sobretudo, exequiível em um período relativamente curto de tempo. Este ideal ainda está longe de ser alcançado (e.g. Gainotti, 2006). Neste estudo nós procuramos, portanto, contribuir para caracterizar a heterogeneidade neuropsicológica da EM e as características clinicas e sócio-demográficas associadas.

O objetivo deste estudo é verificar se o funcionamento cognitivo e psicossocial de uma amostra de portadores de EM e de uma amostra de controles recrutados na população sadia pode contribuir para formar grupos mais homogêneos do ponto de vista neuropsicológico. Nesse sentido, optou-se pela análise de conglomerados, técnica estatística multivariada cujo objetivo principal é agrupar objetos a partir das características que possuem (Everitt, 1993; Hair, Anderson, Tatham, \& Black, 1998). Em um segundo momento, investigou-se as eventuais diferenças quanto aos perfis neuropsicológicos, características clínicas e sócio-demográficas dos conglomerados obtidos. A análise de variância (ANOVA One-Way) e o método de comparações múltiplas post hoc foram utilizados para esse objetivo. Finalmente, tendo em vista a influência da idade e escolarização formal sobre o funcionamento cognitivo, foi utilizada a análise de covariância (ANCOVA), controlando estas variáveis na comparação entre os conglomerados.

\section{Amostra}

\section{Características Sócio-Demográficas dos Participantes}

Oitenta e quatro indivíduos foram recrutados para o estudo. Entretanto, como o método utilizado na análise de conglomerados é sensível à presença de outliers, estes foram identificados através de gráficos de caixas ("boxplots") e retirados previamente. Assim, quatro pessoas saudáveis foram excluídas das análises, reduzindo a amostra total para 80 participantes. Após os ajustes mencionados, o grupo de pacientes portadores de esclerose múltipla permaneceu composto por 35 indivíduos. Quarenta e cinco pessoas constituíram o grupo controle.

A média de idade dos pacientes foi de $42.09(D P=8.93)$ anos, sendo que o percentual de pessoas do sexo feminino foi $65,7 \%$. A escolaridade formal média foi igual a 10.74 $(D P=4.55)$ anos.

A média de idade do grupo controle foi igual a 36.27 $(D P=14.01)$ anos com escolaridade média de 11.96 $(D P=3.36)$ anos. A freqüência relativa de pessoas do sexo feminino foi de $77.8 \%$. Contudo, não houve diferenças significativas entre as duas amostras nas variáveis citadas.

\section{Instrumentos de Coleta de Dados}

Os instrumentos utilizados no presente estudo foram selecionados previamente a partir de dois critérios: evidências de comprometimento neuropsicológico em relação aos construtos avaliados descritos na literatura (Thornton \& Raz, 1997; Wishart \& Sharpe, 1997; Zakzanis, 2000) e 
acurácia diagnóstica a partir do método de análise de características receptivo-operatórias (análise ROC, Swets, 1988). O método citado foi previamente aplicado a 11 instrumentos de avaliação neuropsicológica, sendo 9 relacionados à avaliação cognitiva e 2 relacionados à avaliação psicossocial (Lima, 2005). Não foi verificada a acurácia diagnóstica das escalas de avaliação neurológica, pois estas não foram aplicadas no grupo controle e não foram consideradas na análise de conglomerados. Entre as medidas de avaliação neuropsicológica, considerou-se para o presente estudo apenas as medidas com acurácia moderada ou alta (Swets, 1988).

Visando facilitar a apresentação dos instrumentos utilizados no presente estudo, é possível dividi-los em três categorias: (a) escalas de avaliação neurológica; (b) instrumentos de avaliação cognitiva; e (c) questionários de avaliação psicossocial.

\section{Escalas de Avaliação Neurológica.}

Expanded Disability Status Scale - EDSS: A EDSS (Kurtzke, 1983) é uma escala ordinal que se baseia no exame neurológico de oito sistemas funcionais: (a) funções piramidais, relacionadas ao funcionamento motor; (b) funções cerebelares, relacionadas à coordenação de movimentos; (c) funções do tronco cerebral, relacionadas a movimentos oculares, coordenação da fala e deglutição; (d) funções vesicais e intestinais, relacionada ao controle dos esfíncteres; (e) funções sensoriais, relacionadas à sensibilidade e propriocepção; (f) funções visuais; (g) funções mentais, relacionadas ao humor e à cognição; e (h) outras funções ligadas a achados neurológicos compatíveis com a EM. A EDSS é útil principalmente como um instrumento para classificar pacientes em função da severidade do comprometimento neurológico e como um método para avaliação do grau de incapacidade funcional.

Índice Ambulatorial: O Índice de Ambulação ou Ìndice Ambulatorial (IA), desenvolvido por Hauser et al. (1983), é uma forma simplificada para se obter uma estimativa global do nível de incapacidade física em portadores de esclerose múltipla. O instrumento avalia de forma clínica e semi-quantitativa a capacidade de deambulação dos pacientes.

\section{Instrumentos de Avaliação Cognitiva.}

Bateria de Avaliação da Memória de Trabalho (BAMTUFMG): A BAMT-UFMG é um procedimento de lápis e papel que avalia a memória de trabalho conforme um modelo de três processos desenvolvido por Salthouse e Babcock (1991): eficácia de processamento, armazenamento e função executiva. O instrumento é constituído de dois conjuntos de três tarefas homólogas empregando estímulos numéricos e verbais e medindo cada um dos três construtos do modelo de memória de trabalho (vide Wood, Carvalho, Rothe-Neves \& Haase, 2001; Wood et al., 2000). Cada versão consiste de três testes: (a) de velocidade de processamento, avaliada através do número de problemas aritméticos e respostas corretas sobre frases dentro de um limite fixo de tempo; (b) de armazenamento, que consiste em medir o alcance de apreensão de listas de dígitos e de palavras; e (c) de coordenação de operações, que envolve a execução simultânea de duas tarefas: resolver problemas aritiméticos e armazenar dígitos e responder perguntas sobre frases e armazenar palavras.

Teste de Fluência Verbal: O teste de fluência verbal possui algumas variações em sua aplicação, contudo, o modelo normalmente adotado consiste em solicitar ao paciente que gere em 60 segundos o maior número possível de itens pertencentes a um determinado tipo sem repetição (vide Spreen \& Strauss, 1998). No presente estudo, utilizou-se a versão com palavras iniciadas com determinada letra $(\mathrm{F}$, A ou S), versão também conhecida como fluência verbal ortográfica.

Teste dos Trigramas Consonontais: O procedimento para o teste dos trigramas consonontais avalia os processos de interferência na memória verbal de curto-prazo. O teste avalia também a atenção dividida e a capacidade de processamento de informação, podendo ser considerado uma medida do processamento executivo central. O procedimento consiste em solicitar ao examinado que repita uma série de trigramas consonantais (p.ex., XTN, STJ. etc.) após a realização de uma tarefa distratora (Lezak, 1995, Spreen \& Strauss, 1998).

\section{Questionários de Avaliação Psicossocial.}

Questionário de Saúde Geral de Goldberg: O Questionário de Saúde Geral de Goldberg - QSG (Pasquali, Gouveia, Andriola, Miranda \& Ramos, 1996) foi utilizado na avaliação de sintomas psicopatológicos em portadores de esclerose múltipla. O QSG é um instrumento que objetiva fornecer uma perspectiva de pacientes não-psicóticos sobre o seu estado de saúde mental, o qual foi validado para o Brasil com excelentes características psicométricas, além de ter sido amplamente empregado em pesquisas com portadores de esclerose múltipla (Dalos, Rabins, Brooks \& O’Donnel, 1983, Feinstein \& Feinstein, 2001, Haase et al., 2004). O QSG consiste em 60 itens, aferidos conforme uma escala com 4 graus, que varia de 1 - "não absolutamente", 2 - "não mais do que de costume", 3 - "um pouco mais do que de costume" até 4 - "muito mais do que de costume". Além do escore original, os resultados do QSG originam 5 escores parciais relacionados à presença de estresse, desejo de morte, desconfiança no próprio desempenho, distúrbios do sono e distúrbios psicossomáticos.

Inventário Beck para depressão: Com o intuito de avaliar a presença de sintomas depressivos foi selecionado o Inventário Beck para Depressão - IBD (Beck, Ward, Mendelson, Mock \& Erbaugh, 1961). A escolha deste instrumento se justifica tanto pelo fato de haver dados recentes quanto à sua validação para a população brasileira (Gorenstein \& Andrade, 1998), quanto pela existência de dados de pesquisa com a utilização do IBD no Brasil em amostras de portadores de esclerose múltipla, o que permitirá a realização de comparações (Andrade et al., 1999, Haase et al., 2004). 


\section{Resultados}

Características Clinicas dos Portadores de EM

A média para a duração da doença foi igual a 9.58 $(D P=7.64)$ anos. A idade média de início da doença foi 31.66 $(D P=10.1)$ anos. O IA médio foi igual a $2.0(D P=1.82)$ e o EDSS igual a 3.02.( $D P=1.96)$. Setenta e dois por cento dos pacientes apresentaram a forma recorrente-remitente da doença.

\section{Análise de Conglomerados}

Previamente à análise de conglomerados, optou-se pela transformação dos escores brutos em escores padronizados, pois houve uma diferença significativa entre os desvios padrões, fator importante a ser considerado nos passos seguintes da análise.

Os escores brutos foram transformados em resultados percentílicos. A normatização dos dados foi calculada a partir da maior pontuação obtida entre os participantes nos diferentes instrumentos. Nos testes de avaliação cognitiva, onde quanto maior o escore, melhor o desempenho da pessoa, a fórmula utilizada foi: (100 - escore individual / maior escore obtido entre os participantes da amostra). Por outro lado, nos questionário de avaliação psicossocial, onde quanto maior o escore, pior o desempenho do participante, a fórmula utilizada foi: [100 - (100 escore individual / maior escore obtido entre os participantes da amostra)]. Dessa forma, os escores sempre variaram de $\mathrm{O}$ a 100 e manteve-se constante a lógica de que quanto maior a pontuação melhor o desempenho, independente do instrumento em questão.

De acordo com Hair et al. (1998), variáveis com coeficientes de correlação moderados a altos acarretam complicações nas análises devido à redundância dos fatores avaliados. Nesses casos, é aconselhável retirar uma das variáveis em questão da análise de conglomerados a ser realizada. As correlações realizadas foram estatisticamente significativas, variando de intensidade baixa a moderada. O coeficiente de correlação encontrado entre o Questionário de Saúde Ge- ral (fator Saúde Geral) e o Inventário Beck para Depressão, indica que os instrumentos são incompatíveis para uma mesma análise de conglomerados (rho = 0.765; $p<0.001$ ) Contudo, optou-se por incluir os dois instrumentos, considerando que os construtos avaliados são distintos e devido à importância atribuída aos sintomas depressivos na EM (Arnett et al., 1999; Haase et al., 2004). Os outros coeficientes de correlação encontrados não sugerem dificuldades para a análise de conglomerados.

Em relação à estratégia de formar grupos, escolheu-se o método de Ward, um método hierárquico, o que se justifica pela facilidade e ausência de necessidade de estabelecer pontos centrais, pela sua eficácia para amostras compostas por ruídos (Everitt, 1993), bem como pelo caráter heterogêneo do comprometimento neuropsicológico na EM (Amato, Zipoli \& Portaccio, 2006). Métodos como o de ligação simples ou ligação completa também foram conduzidos previamente, mas foram observados efeitos de "encadeamento" (Everitt), mascarando a presença de subgrupos. Finalmente, foi escolhido o método da distância euclidiana ao quadrado, que é o procedimento mais adequado para o método de Ward (Everitt).

A análise de conglomerados resultou em uma solução ideal com cerca de 4 conglomerados. Ao realizar o $78^{\circ}$ passo ou fusão, o coeficiente sofreu um salto significativamente superior aos precedentes. Essa diferença indica um aumento brusco da heterogeneidade intragrupo, indicando o momento de interromper a análise. A seguir, os resultados serão interpretados teoricamente.

\section{Perfil Neuropsicológico dos Conglomerados Formados}

A solução explorada mais detalhadamente na análise foi decorrente do $77^{\circ}$ passo, resultando em 4 grupos. Em relação ao perfil neuropsicológico, os conglomerados diferiram de forma nítida nas medidas utilizadas na análise. As características apresentadas abaixo estão de acordo com a heterogeneidade do perfil neuropsicológico na esclerose múltipla. A Tabela 1 apresenta o perfil dos quatro conglomerados.

Tabela 1

Perfil Neuropsicológico dos Grupos Encontrados na Análise de Conglomerados

\begin{tabular}{llllll}
\hline & & Grupo 1 & Grupo 2 & Grupo 3 & Grupo 4 \\
& $n$ & 25 & 13 & 30 & 12 \\
\hline QSG - Saúde Geral & Média & 53.10 & 50.58 & 37.60 & 23.30 \\
& $(D P)$ & $(5.13)$ & $(5.97)$ & $(11.71)$ & $(13.85)$ \\
Inventário Beck & Média & 92.45 & 83.95 & 63.61 & 31.66 \\
& $(D P)$ & $(8.16)$ & $(5.65)$ & $(15.41)$ & $(16.81)$ \\
Velocidade de Processamento & Média & 61.53 & 36.65 & 50.02 & 28.62 \\
Fluência Verbal & $(D P)$ & $(13.08)$ & $(10.47)$ & $(8.64)$ & $(13.49)$ \\
& Média & 64.18 & 43.24 & 58.63 & 38.76 \\
Trigramas Consonontais & $(D P)$ & $(11.69)$ & $(10.26)$ & $(12.34)$ & $(12.23)$ \\
& Média & 86.44 & 62.45 & 75.31 & 49.43 \\
& $(D P)$ & $(9.23)$ & $(15.51)$ & $(11.27)$ & $(11.33)$
\end{tabular}


A análise de conglomerados apontou uma solução ideal onde as medidas consideradas podem ser agrupadas conforme duas dimensões de comprometimento, respectivamente, cognitivo e psicossocial. A Figura 1 apresenta os resultados obtidos nos instrumentos de avaliação psicossocial e a Figura 2 apresenta os resultados obtidos nos testes de avaliação cognitiva. Ambas as figuras foram construídas em função aos quatro conglomerados obtidos usando as duas dimensões

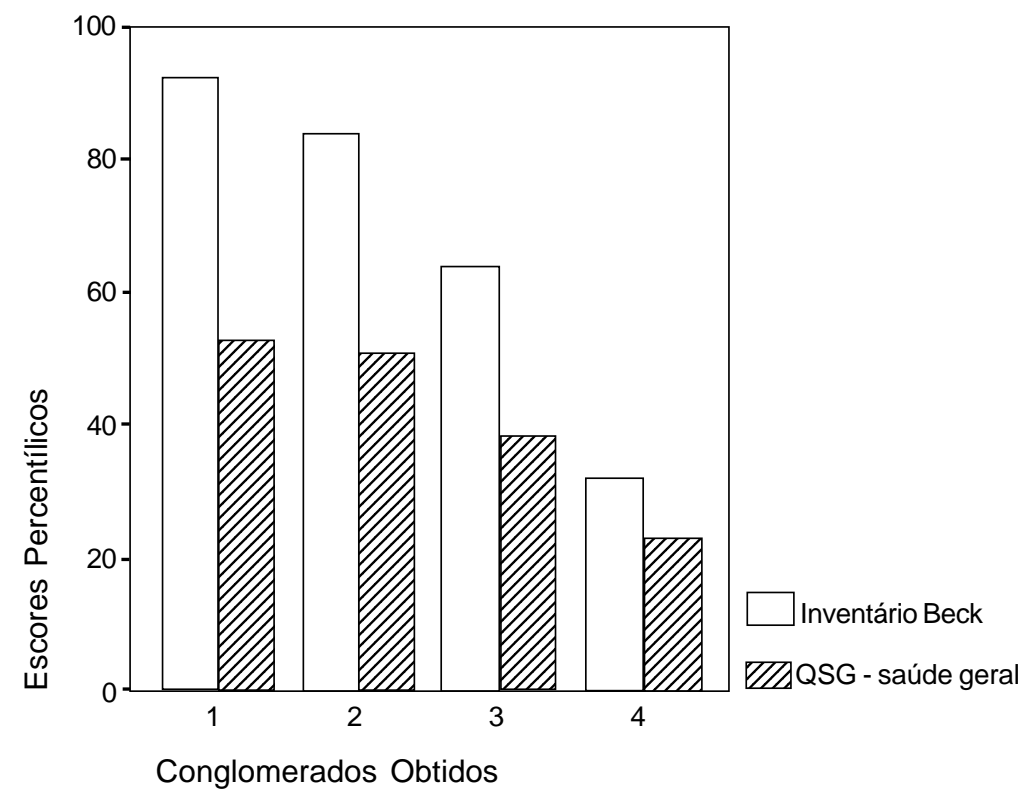

Figura 1. Desempenho dos conglomerados nos questionários de avaliação psicossocial

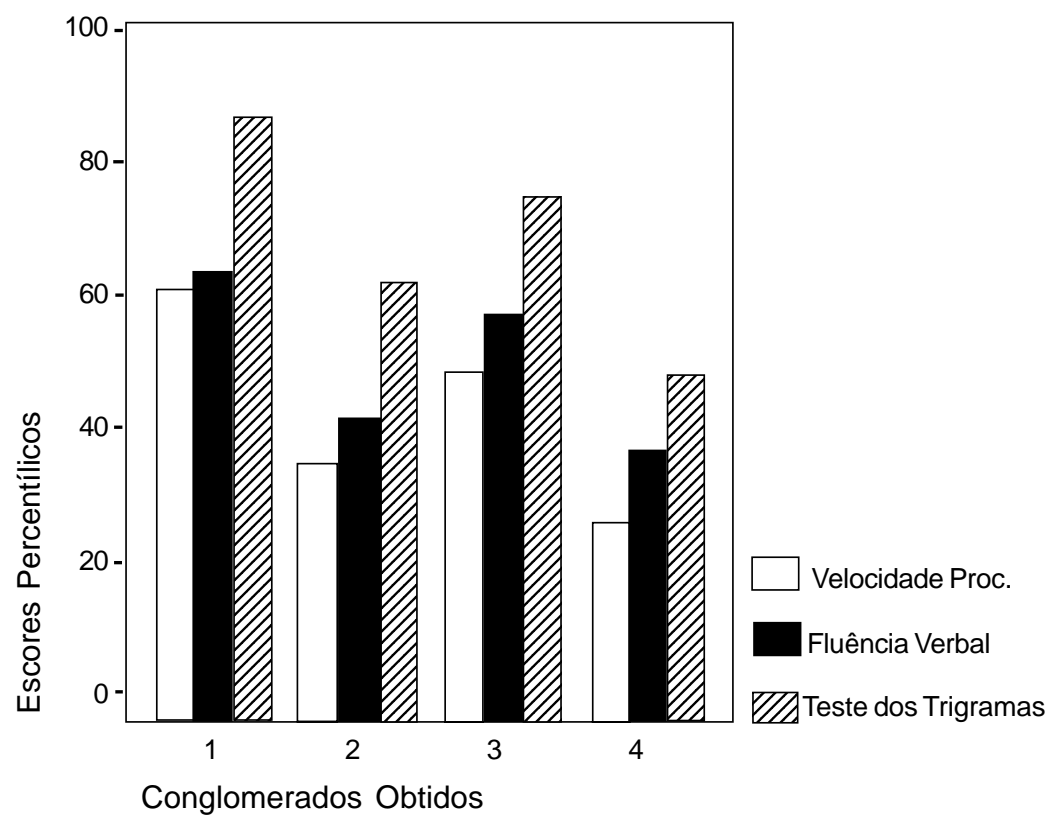

Figura 2. Desempenho dos conglomerados nos instrumentos de avaliação cognitiva

O primeiro grupo apresenta um perfil cognitivo e psicossocial superior aos outros conglomerados. O último grupamento, por sua vez, apresenta um desempenho deficitário em ambas as dimensões. O segundo e o terceiro grupamento apresentam um desempenho intermediário em relação ao perfil neuropsicológico. Contudo, a dife- rença entre eles sugere uma dissociação entre perfil cognitivo e perfil psicossocial. O grupo 2 apresenta um desempenho cognitivo inferior, próximo ao desempenho do grupo com o pior desempenho, mas apresenta um funcionamento psicossocial adaptado, semelhante ao grupo 1 em relação à presença de sintomas de depressão e de ou- 
tras psicopatologias. O grupo 3 apresenta um resultado inverso. Os escores desse conglomerado nos três instrumentos de avaliação cognitiva foram bastante próximos dos valores apresentados pelo grupo 1, contudo o perfil psicossocial parece prejudicado.

Os conglomerados foram analisados também quanto à freqüência de participantes que ultrapassaram os pontos de cor te estabelecidos para diagnósticos psicopatológicos. Nos conglomerados 1 e 2 nenhum participante apresentou desempenho acima dos pontos de corte. No conglomerado 3, 67\% dos participantes apresentaram sintomas depressivos leves no IBD (escores entre 11 e 20) a moderados (escores entre 21 e 25) conforme os critérios de Beck et al. (1961). Em comparação, todos os participantes agrupados no conglomerado 4 apresentaram sintomas depressivos, sendo 33,3\% com sintomatologia depressiva grave (escores acima de 25 pontos). No que se refere ao QSG, 13,3\% dos participantes do conglomerado 3 apresentaram escores superiores ao percentil 90, conforme critérios estabelecidos por Pasquali et al. (1996), enquanto esta freqüência foi igual a $66,7 \%$ no conglomerado 4 .

\section{Perfil Sócio-Demográfico dos Conglomerados Formados}

Em seguida, a partir da divisão em função do perfil neuropsicológico dos participantes, pretendeu-se identificar características sócio-demográficas relacionadas aos resultados. As características consideradas a seguir sugerem possíveis fatores relacionados ao perfil neuropsicológico dos participantes do estudo, além de contribuir para a validação da solução final escolhida. A análise descritiva do perfil sócio-demográfico dos conglomerados foi realizada separadamente para portadores e controles. A Tabela 2 resume os dados apresentados.

Tabela 2

Características Demográficas dos Grupos Encontrados na Análise de Conglomerados

\begin{tabular}{|c|c|c|c|c|c|c|c|c|}
\hline & \multicolumn{2}{|c|}{ Grupo 1} & \multicolumn{2}{|c|}{ Grupo 2} & \multicolumn{2}{|c|}{ Grupo 3} & \multicolumn{2}{|c|}{ Grupo 4} \\
\hline & Média & $(D P)$ & Média & $(D P)$ & Média & $(D P)$ & Média & $(D P)$ \\
\hline$N$-pacientes & \multicolumn{2}{|c|}{4} & \multicolumn{2}{|c|}{6} & \multicolumn{2}{|c|}{13} & \multicolumn{2}{|c|}{11} \\
\hline$N-$ controles & \multicolumn{2}{|c|}{21} & \multicolumn{2}{|c|}{7} & \multicolumn{2}{|c|}{17} & \multicolumn{2}{|c|}{1} \\
\hline \% pacientes EM & \multicolumn{2}{|c|}{$16 \%$} & \multicolumn{2}{|c|}{$46.2 \%$} & \multicolumn{2}{|c|}{$43.3 \%$} & \multicolumn{2}{|c|}{$91.7 \%$} \\
\hline Idade pacientes (anos) & 38.25 & $(7.14)$ & 44.57 & $(8.44)$ & 41.46 & $(9.65)$ & 42.64 & $(9.50)$ \\
\hline Idade controles (anos) & 34.19 & $(12.85)$ & 46.0 & $(10.95)$ & 36.59 & $(9.24)$ & 36.0 & 米米 \\
\hline Escolaridade pacientes (anos) & 11.00 & $(4.08)$ & 10.57 & $(5.22)$ & 13.38 & $(4.03)$ & 7.64 & $(3.11)$ \\
\hline Escolaridade controles (anos) & 13.24 & $(2.46)$ & 7.17 & $(3.87)$ & 12.29 & $(2.64)$ & 8.0 & 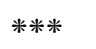 \\
\hline$\%$ sexo fem. (pacientes) & \multicolumn{2}{|c|}{$75 \%$} & \multicolumn{2}{|c|}{$57.1 \%$} & \multicolumn{2}{|c|}{$69.2 \%$} & \multicolumn{2}{|c|}{$60.0 \%$} \\
\hline$\%$ sexo fem. (controles) & \multicolumn{2}{|c|}{$71.4 \%$} & \multicolumn{2}{|c|}{$83 \%$} & \multicolumn{2}{|c|}{$82.4 \%$} & \multicolumn{2}{|c|}{$100 \%$} \\
\hline
\end{tabular}

A distribuição dos participantes dentro dos quatro conjuntos contribui positivamente para a validação do resultado da análise. O grupamento 1 é composto, em sua maioria, por controles (21 pessoas - 84\%), enquanto o grupo 4 é formado por onze pacientes e apenas um controle. Esse resultado demonstra que as variáveis selecionadas para a análise e a solução final obtida foram adequadas e, de fato, existe uma relação entre perfil neuropsicológico e esclerose múltipla.

Entre os pacientes, o grupo 1 apresenta o maior índice de participantes do sexo feminino (75\%). Esse resultado oferece supor te à hipótese de que o sexo feminino é preditor de um prognóstico favorável da doença. Contudo, a percentagem de pessoas do sexo feminino nos outros grupos oferece resultados inconclusivos.

Os conglomerados 2 e 3 não distinguem entre pacientes e controles. Contudo, outras variáveis podem contribuir para esclarecer essa distribuição. Os grupamentos 1 e 3 apresentam as menores médias de idade, tanto para os pacientes quanto para os controles. Esses dois conglomerados apresentaram um desempenho cognitivo semelhante, superior aos outros grupos e a idade é sabidamente uma variável importante para o desempenho cognitivo em testes de inteligência fluída (Salthouse \& Babcock, 1991). O número de anos de escolarização formal, outra variável importante para o funcionamento da cognição, também é o mais elevado para os dois grupos. O conglomerado 4 é composto por pacientes mais velhos e com escolarização mais baixa. O único participante saudável presente nesse conjunto apresenta poucos anos de escolarização formal, possuindo apenas o primeiro grau completo. O grupo 2 apresenta um perfil semelhante ao grupo 4. Os resultados sugerem uma relação estreita entre idade, escolaridade e perfil cognitivo. O funcionamento psicossocial não parece estar relacionado às variáveis idade e escolaridade.

\section{Características Clínicas dos Conglomerados Formados}

Finalmente, são apresentadas as características clínicas para cada um dos grupos encontrados. Considerou-se nessa última análise a forma clínica da esclerose múltipla, a duração (em anos) da doença a partir dos primeiros sintomas, a idade de início da doença e duas medidas de funcionamento neurológico: o Índice Ambulatorial e a EDSS. A Tabela 3 resume os achados. 
Tabela 3

Características Clínicas dos Grupos Encontrados na Análise de Conglomerados

\begin{tabular}{|c|c|c|c|c|c|c|c|c|}
\hline & \multicolumn{2}{|c|}{ Grupo 1} & \multicolumn{2}{|c|}{ Grupo 2} & \multicolumn{2}{|c|}{ Grupo 3} & \multicolumn{2}{|c|}{ Grupo 4} \\
\hline & Média & $(D P)$ & Média & $(D P)$ & Média & $(D P)$ & Média & $(D P)$ \\
\hline$N$ - controle & \multicolumn{2}{|c|}{21} & \multicolumn{2}{|c|}{6} & \multicolumn{2}{|c|}{17} & \multicolumn{2}{|c|}{1} \\
\hline$N$-pacientes & \multicolumn{2}{|c|}{4} & \multicolumn{2}{|c|}{7} & \multicolumn{2}{|c|}{13} & \multicolumn{2}{|c|}{11} \\
\hline$\%$ pacientes EM & \multicolumn{2}{|c|}{$16 \%$} & \multicolumn{2}{|c|}{$46.2 \%$} & \multicolumn{2}{|c|}{$43.3 \%$} & \multicolumn{2}{|c|}{$91.7 \%$} \\
\hline \% Forma Remitente -Recorrente & \multicolumn{2}{|c|}{$66.7 \%$} & \multicolumn{2}{|c|}{$100 \%$} & \multicolumn{2}{|c|}{$63.6 \%$} & \multicolumn{2}{|c|}{$60 \%$} \\
\hline \% Formas Progressivas & \multicolumn{2}{|c|}{$33.3 \%$} & \multicolumn{2}{|c|}{$\mathrm{O} \%$} & \multicolumn{2}{|c|}{$36.4 \%$} & \multicolumn{2}{|c|}{$40 \%$} \\
\hline Idade de Início da doença & 33.75 & $(7.79)$ & 27.33 & $(7.79)$ & 31.08 & $(10.98)$ & 34.10 & $(11.30)$ \\
\hline Duração da Doença & 4.50 & $(3.42)$ & 18.33 & $(9.50)$ & 9.58 & $(8.54)$ & 8.90 & $(6.67)$ \\
\hline Índice Ambulatorial & 1.75 & $(2.36)$ & 2.40 & $(1.67)$ & 1.55 & $(1.92)$ & 2.57 & $(1.62)$ \\
\hline EDSS & 1.50 & $(2.12)$ & 3.00 & $(1.87)$ & 2.85 & $(1.88)$ & 3.64 & $(2.23)$ \\
\hline
\end{tabular}

O grupo 1 é predominantemente composto por pessoas saudáveis. Entre os pacientes do grupo, 66.7\% são portadores da forma remitente-recorrente da doença e $33.3 \%$ portadores de formas crônico-progressivas. A idade média de início da doença é $33.75(D P=7.79)$ anos. Além disso, o grupo apresenta a menor média de duração da doença ( $M=4.50$ anos, $D P=3.42$ anos $)$ e baixos índices de comprometimento físico, de acordo com as escalas utilizadas.

O grupamento 2 é composto por controles e pacientes de forma equilibrada. Entre os pacientes, $100 \%$ são portadores da forma remitente-recorrente da doença. O grupo apresentou a menor média de idade de início dos sintomas da esclerose múltipla ( $M=27.33$ anos; $D P=7.79$ anos) e maior média de duração da doença $(M=18.33$ anos; $D P=9.50$ anos). Nas escalas de incapacidade física, os resultados indicam índices de comprometimento físico moderados.

O grupo 3 também é composto por pacientes e controles de forma equilibrada. Entre os pacientes, 63.6\% apresentam a forma remitente-recorrente da doença e $36.4 \%$ formas progressivas. A idade média de início da doença ( $M=31.08$ anos; $D P=10.98$ anos) e a média de duração da doença ( $M=9.58$ anos; $D P=8.54$ anos) indicam valores intermediários entre os conglomerados. Esse grupo também apresenta o menor índice de comprometimento físico de acordo com o Índice Ambulatorial, uma das escala utilizadas. Entretanto, o escore médio na EDSS foi próximo ao conglomerado com maior comprometimento neuropsicológico $(M=2.85 ; D P=1.88)$.

Finalmente, o grupo 4 é composto, em sua maioria, por pacientes portadores de esclerose múltipla. Este grupo apresenta o maior índice de pacientes portadores das formas progressivas da doença $(40 \%)$, achado que oferece suporte à hipótese de que as formas progressivas acarretam um comprometimento neuropsicológico de maior magnitude. Sessenta por cento dos pacientes do terceiro conglomerado apresentam a forma remitente recorrente. O grupo apresenta a maior média de idade de início da doença $(M=34.10$ anos; $D P=11.30$ anos). A média de du- ração da doença é $8.9(D P=6.67)$ anos. Os índices de comprometimento físico foram os mais acentuados entre os quatro conglomerados.

\section{Análise de Variância (ANOVA One-Way) e Comparações Múltiplas}

Foram realizadas análises de variância (ANOVA OneWay) e comparações múltiplas utilizando o método de Bonferroni objetivando identificar diferenças significativas entre os quatro conglomerados formados para: (a) instrumentos de avaliação neuropsicológica, (b) características sócio-demográficas, e (c) parâmetros da doença.

Em relação à avaliação neuropsicológica, houve diferenças significativas entre os grupos para as cinco variáveis utilizadas na análise $\left(\mathrm{F}_{\mathrm{s}[3,76]}\right.$ variando entre 17,814 e 71,663, todos a $p<.001$ ). Análises de covariância (ANCOVA) indicaram que a diferença entre os grupos permaneceu significativa para os três testes de avaliação cognitiva mesmo após o controle das variáveis idade e escolaridade. As comparações múltiplas realizadas demonstram diferenças nítidas entre os grupos, oferecendo suporte ao resultado final obtido pela análise de conglomerados. Nos instrumentos de avaliação cognitiva, os resultados indicaram diferenças significativas entre os grupos 1 e 2 (todos a $p<.001$ ), 1 e 3 (Velocidade de Processamento $-p=.004$ e Trigramas Consontais $-p=.008), 1$ e 4 (todos a $p<.001)$ e 3 e 4 (todos a $p<.001$ ). Por outro lado, na avaliação do funcionamento psicossocial, foram observadas diferenças para o fator saúde geral do QSG entre todos os grupos, exceto entre os conglomerados 1 e 2. Todas as comparações foram significativas para o Invetário Beck para Depressão. Apesar das diferenças significativas em quase todas as comparações post hoc, os achados confirmam a hipótese de dissociação entre o perfil cognitivo e psicossocial, considerando as diferenças mais significativas obedecem ao padrão observado nas Figuras 1 e 2 .

Os resultados das análises de variância (ANOVA One-Way) para as variáveis sócio-demográficas indicam diferenças significativas entre os conglomerados para as variáveis escolarização formal $\left(\mathrm{F}_{[3,76]}=10,203, p<0.001\right)$ 
e idade $\left(\mathrm{F}_{[3,76]}=3,278, p=0.025\right)$. As diferenças, entre os grupos, relacionadas à escolarização formal seguiu o mesmo padrão do funcionamento cognitivo, reforçando a afirmação feita a respeito da estreita correlação entre as duas variáveis. O número de anos de escolarização formal foi significativamente distinto entre os grupos 1 e 2 ( $p=.007)$, 1 e $4(p<.001), 2$ e $3,(p=.007)$ e 3 e $4(p<.001)$. Em relação à idade, as comparações evidenciaram diferenças entre os grupos 1 e $2(p=0.02)$.

Finalmente, os resultados das análises de variância (ANOVA One-Way) para os parâmetros da doença indicam diferenças significativas entre os conglomerados para as variáveis amostra $\left(\mathrm{F}_{[3,76]}=8,198, p<0.001\right)$ e duração da doença $\left(\mathrm{F}_{[3,76]}=3,023, p=0.046\right)$. As comparações múltiplas utilizando o método de Bonferroni mostram que em relação à amostra, houve diferenças significativa entre os grupos 1 e $4(p<.001)$ e 3 e $4(p=.002)$. Esses resultados indicam que o conglomerado 4 pode, de fato, ser nomeado como grupo clínico. Os grupos intermediários 2 e 3, como mencionado anteriormente, não distinguem pacientes de controles. O grupo 1, por outro lado, é composto predominantemente por pessoas saudáveis. Diferenças significativas na variável "duração da doença" foram observadas apenas na comparação dos dois primeiros conglomerados $(p=0.047)$.

\section{Conclusão e Discussão}

\section{Interpretação dos Conglomerados Obtidos}

A esclerose múltipla acarreta comprometimento em diferentes aspectos do funcionamento neuropsicológico (Thornton \& Raz, 1997; Wishart \& Sharpe, 1997; Zakzanis, 2000). A heterogeneidade das manifestações é uma característica peculiar da doença. Alguns pacientes permanecem sem comprometimentos importantes durante anos (Amato, Zipoli, \& Portaccio, 2006). Por outro lado, outras pessoas apresentam déficits cognitivos, motores e comprometimento psicossocial de magnitude alta já nos primeiros anos de evolução da esclerose múltipla (O'Connor, 2002). Na literatura neuropsicológica, diversos fatores têm sido apontados com o intuito de explicar a amplitude desta variação: a duração e a idade de início da doença, a forma clínica da esclerose múltipla, o número de lesões observadas em procedimentos de neuro-imagem, a idade, o número de anos de escolarização formal e outros fatores relacionados ao status econômico e social dos indivíduos (Zakzanis, 2000).

Como reflexo da heterogeneidade neurológica e psicológica observada na EM, os quatro grupamentos obtidos na análise mostraram perfis bastante diferenciados. Diante dos resultados descritos, sugere-se a seguinte interpretação para cada grupo:

Grupo 1 - Saudável: esse primeiro grupo, composto predominantemente por pessoas saudáveis (84\%), apresentou ausência de comprometimento nos questionários de avaliação psicossocial e os melhores desempenhos na avaliação cognitiva.
Grupo 2 - Baixo desempenho cognitivo: o grupo 2 é composto por pacientes e controles saudáveis em proporções aproximadamente iguais. Esse conglomerado apresentou um nível de funcionamento cognitivo mais baixo, com desempenhos inferiores aos grupos 1 e 3 nos três instrumentos aplicados. O perfil psicossocial não diferiu do "Grupo Saudável", tal como avaliado pelo QSG. Nenhum participante desse grupamento apresentou sintomas psicopatológicos no QSG ou níveis patológicos de sintomática depressiva no IBD.

Grupo 3-Comprometimento psicossocial: o grupo três também não distinguiu entre pacientes e controles. O conglomerado apresentou um desempenho cognitivo satisfatório, mais próximo do perfil apresentado pelo Grupo 1. Entretanto, dois terços dos sujeitos apresentaram sintomática depressiva e psicopatológica não-psicótica, com diferenças significativas quando comparado com os conglomerados 1 e 2 . Além disso, 26 pessoas (86\%) relataram sintomas depressivos em algum grau.

Grupo 4-Comprometimento global: o quarto grupo é composto por 11 portadores de esclerose múltipla $(91,7 \%)$ e apenas um controle. Esse conglomerado apresentou prejuízos nas duas dimensões avaliadas. A magnitude dos desempenhos nos testes e questionários foi bastante inferior, apresentando diferenças significativas em relação aos grupos 1 e 3, em todos os instrumentos. Todos os participantes apresentaram sintomas depressivos. Entre os pacientes, o conglomerado 4 apresentou a maior percentagem de pessoas com as formas progressivas da doença (40\%), achado que oferece suporte à hipótese de que as formas progressivas acarretam um comprometimento neuropsicológico de maior magnitude.

Um aspecto importante observado a partir da análise de conglomerados é a independência entre os dois domínios de funcionamento. Os resultados apresentados sugerem que há uma dissociação entre os comprometimentos cognitivo e psicossocial. Contudo, apesar da independência entre domínios, estes não são disjuntivos, na medida em que alguns grupos encontrados apresentam desempenhos satisfatórios em ambas as dimensões, enquanto outros apresentam resultados deficitários para estas mesmas funções.

Em relação às variáveis sócio-demográficas e aos parâmetros da doença, os resultados sugerem uma ligação entre funcionamento cognitivo, idade e escolarização formal. Contudo, outros aspectos foram inconclusivos.

$\mathrm{O}$ estudo relatado sugere que diferentes aspectos do funcionamento neuropsicológico podem estar comprometidos na esclerose múltipla. A heterogeneidade da amostra estudada reflete a mesma característica da doença e a imprevisibilidade do curso clínico. As conclusões do estudo apresentam implicações clínicas e psicossociais. A esclerose múltipla é uma doença bastante imprevisível do ponto de vista de sua evolução e os comprometimentos físico, cognitivo e psicossocial estão interligados, afetando o funcionamento psicossocial dos portadores em diferentes graus. Os resultados apresentados indicam, entre- 
tanto, que as diferentes dimensões citadas são independentes, apesar de interligadas e podem ser utilizadas, junto com critérios clinico-neurológicos, para definir perfis de comprometimento na esclerose múltipla. Os perfis identificados, respectivamente a ausência de comprometimento, os comprometimentos predominantemente psicossocial ou cognitivo, e o comprometimento global podem representar tipos ideais ou fenótipos neuropsicológicos da EM. Mais estudos são necessários para elucidar a validade de tais tipos, bem como sua associação com diferentes cursos evolutivos da doença. Por exemplo, o comprometimento cognitivo acentuado pode acarretar um risco maior de prejuízo às outras duas funções? Alguns estudos têm demonstrado, por outro lado que, apesar de constituírem dimensões independentes de comprometimento, os sintomas depressivos podem agravar as dificuldades cognitivas (Arnett et al., 1999).

A caracterização de diferentes fenótipos neuropsicológicos na EM levanta questões quanto às correlações estrutura-função dos sintomas observados na doença, os quais não podem ser explicados com base em modelos localizacionistas clássicos ou estritos. As lesões observadas na EM são multifocais ou confluentes, coexistindo em um determinado momento lesões em vários estágios de evolução/regeneração. As lesões comprometem, entre outras, as fibras de substância branca responsáveis pela conectividade e integração funcional córtico-cortical. Gainotti (2006) sugeriu que os perfis de comprometimento neuropsicológico na EM poderiam ser explicados em termos de um modelo de desconexões múltiplas, segundo o qual a interrupção em múltiplos loci de circuitos córticosubcórtico-corticais prejudicaria o funcionamento em domínios que requerem a atividade coordenada de amplas áreas de tecido cerebral, como é o caso da memória episódica e das funções executivas, além de contribuírem para a lentificação do processamento de informação. Calabrese (2006), por outro lado, propôs um modelo de limiar para explicar a expressão do comprometimento cognitivo na EM. Segundo o modelo de limiar, inicialmente as lesões características da doença podem não apresentar repercussões muito significativas do ponto de vista neuropsicológico, tanto devido ao seu pequeno número quanto aos mecanismos de regeneração axonal. Com o evoluir da doença, entretanto, os mecanismos de neuroplasticidade começam a se esgotar, o número de lesões cicatriciais aumenta e as mesmas começam a confluir. Ultrapassado, portanto, um determinado limiar a carga lesional repercute no exame neuropsicológico (Benedict, Caroni \& Bakshi, 2004). Desta forma, os resultados obtidos são encorajadores no sentido de apontarem para a possibilidade de desvendar uma lógica subjacente à heterogeneidade de comprometimento neuropsicológico na EM. A corroboração das hipóteses levantadas dependerá, contudo, em última análise da utilização de técnicas de neuroimagem, estrutural e funcional.

\section{Referências}

Amato, M. P., Zipoli, V., Goretti, B., Portaccio, E., De Caro, M. F., Ricchiuti, L. et al. (2006). Benign multiple sclerosis: Cognitive, psychological and social aspects in a clinical cohort. Journal of Neurology, 253, 1054-1059.

Amato, M. P., Zipoli, V., \& Portaccio, E. (2006). Multiple sclerosisrelated cognitive changes: A review of cross-sectional and longitudinal studies. Journal of the Neurological Sciences, 245, 41-46.

Andrade, V. M., Bueno, O. F. A., Oliveira, M. G. M., Oliveira, A. S. B., Oliveira, E. M. L., \& Miranda, M. C. (1999). Cognitive profile of patientes with relapsing remitting multiple sclerosis. Arquivos de Neuro-Psiquiatria, 57, 775-783.

Arnett, P. A., Higginson, C. I., Voss, W. D., Bender, W. I., Wurst, J. M., \& Tippin, J. M. (1999). Depression in multiple sclerosis: Relationship to working memory capacity. Neuropsychology, 13, 546-556.

Beck, A. T., Ward, C. H., Mendelson, M., Mock, J., \& Erbaugh, J. (1961). An inventory for measuring depression. Archives of General Psychiatry, 4, 53-63.

Benedict, R. H. B., Carone, D. A., \& Bakshi, R. (2004). Correlating brain atrophy with cognitive dysfunction, mood disturbances, and personality disorder in multiple sclerosis. Journal of Neuroimaging, 14, 36-45.

Calabrese, P. (2006). Neuropsychology of multiple sclerosis: An overview. Journal of Neurology, 253(1), 10-15.

Dalos, N. P., Rabins, P. V., Brooks, B. R., \& O'Donnel, P. (1983). Disease activity and emotional state in multiple sclerosis. Annals of Neurology, 13, 573-577.

Everitt, B. (1993). Cluster analysis. New York: Halsted Press.

Feinstein, A., \& Feinstein, K. (2001). Depression associated with multiple sclerosis: Looking beyond diagnosis to sympton expression. Journal of Affective Disorders, 66, 193-198.

Gainotti, G. (2006). Measures of cognitive and emotional changes in multiple sclerosis and underlying models of brain dysfunctional. Journal of Neurological Sciences, 245, 15-20.

Gorenstein, C., \& Andrade, L. (1998). Inventário de depressão de Beck: Propriedades psicométricas da versão em português. Revista de Psiquiatria Clínica, 25, 245-250.

Haase, V. G., Lacerda, S. S., Lima, E. P., Corrêa, T. D., Brito, D. C. S., \& Lana-Peixoto, M. A. (2004). Avaliação do funcionamento psicossocial na esclerose múltipla. Arquivos de NeuroPsiquiatria, 62(2-A), 282-291.

Hair, J. F., Anderson, R. E., Tatham, R. L. \& Black, W. C. (1998). Multivariate data analysis ( $5^{\text {th }}$ ed.). New York: Prentice Hall Press.

Hauser, S. L., Dawson, D. M., Lehrich, J. R., Beal, M. F., Kevy, S. V., Propper, R. D., Mills, J. A., \& Weiner, H. L. (1983). Intensive immunosuppression in progressive multiple sclerosis: A randomized, three-arm study of high-doese intravenous cyclophosphamide, plasma exchange and ACTD. New England Journal of Medicine, 308, 173-180.

Kurtzke, J. F. (1983). Rating neurologic impairment in multiple sclerosis: An expanded disability status scale (EDSS). Neurology, 33, 1444-1452.

Lezak, M. D. (1995). Neuropsychological Assessment. New York: Oxford University Press.

Lima, E. P. (2005). Heterogeneidade do perfil neuropsicológico na esclerose múltipla. Dissertação de Mestrado não-publicada, Universidade Federal de Minas Gerais, Belo Horizonte, MG. Lublin, F., \& Reingold, S. C. (1996). Defining the clinical course of multiple sclerosis: Results of an international survey. Neurology, 44, 907-911. 
McIntosh-Michaelis, S. A., Roberts, M. H., Wilkinson, S. M., Diamond, I. D., Mclellan, D. L., Martin, J. P et al. (1991). The prevalence of cognitive impairment in a community survey of multiple sclerosis. British Journal of Clinical Psychology, 30, 333-348.

O'Connor, P. (2002). Key issues in the diagnosis and treatment of multiple sclerosis: An overview. Neurology, 59, 1-33.

Pasquali, L., Gouveia, V. V., Andriola, W. B., Miranda, F. J., \& Ramos, A. L. (1996). Questionário de saúde geral de Goldberg. Manual Técnico: Adaptação brasileira. São Paulo, SP: Casa do Psicólogo.

Pujol, J., Bello, J., Deus, J., Cardoner, N., Martí-Vilalta, J. L., \& Capdevila, A. (2000). Beck Depression Inventory factors related to demyelinating lesions of the left arcuate fasciculus region. Psychiatry Research, 99, 151-159.

Rao, S. M. (1986). Neuropsychology of multiple sclerosis: A critical review. Journal of Clinical and Experimental Neuropsychology, 8, 503-542.

Rocca, M. A., Colombo, B., Falini, A., Ghezzi, A., Martinelli, V., Scotti, G. et al. (2005). Cortical adaptation in patients with MS: A cross-sectional functional MRI study of disease phenotypes. Lancet Neurology, 4, 618-626.

Salthouse, T. A., \& Babcock, R. L. (1991). Decomposing adult age differences in working memory. Developmental Psychology, 27, 763-776.

Spreen, O., \& Strauss, E. (1998). A compendium of neuropsychological test. Administration, norms and commentary (2. ed). New York: Oxford University Press.

Swets, J. A. (1988). Measuring the accuracy of diagnostic systems. Science, 240, 1285-1293.

Thornton, A. E., \& Raz, N. (1997). Memory impairment in multiple sclerosis: A quantitative review. Neuropsychology, 11, 357-366.

Wishart, H., \& Sharp, D. (1997). Neuropsychological aspects of multiple sclerosis: A quantitative review. Journal of Clinical and Experimental Neuropsychology, 19, 810-857.

Wood, G. M. O., Carvalho, M. R. S., Rothe-Neves, R., \& Haase, V. G. (2001). Validação da bateria de avaliação da memória de trabalho (BAMT-UFMG). Psicologia: Reflexão e Crítica, 14, 763-776.

Wood, G. M. O., Haase, V. G., Araujo, J. R., Lima, E. P., Scalioni, I. G., \& Sampaio, J. R. (2000). Desenvolvimento cognitivo adulto: A avaliação e a reabilitação da capacidade de memória de trabalho. In V. G. Haase, R. Rothe-Neves, C. Käppler, M. L. M. Teodoro \& G. M. O. Wood (Eds.), Psicologia do desenvolvimento: Contribuições interdisciplinares (pp. 121-144). Belo Horizonte, MG: Health.

Zakzanis, K. K. (2000). Distinct neurocognitive profiles in multiple sclerosis subtypes. Archives of Clinical Neuropsychology, 15, 115-136. 